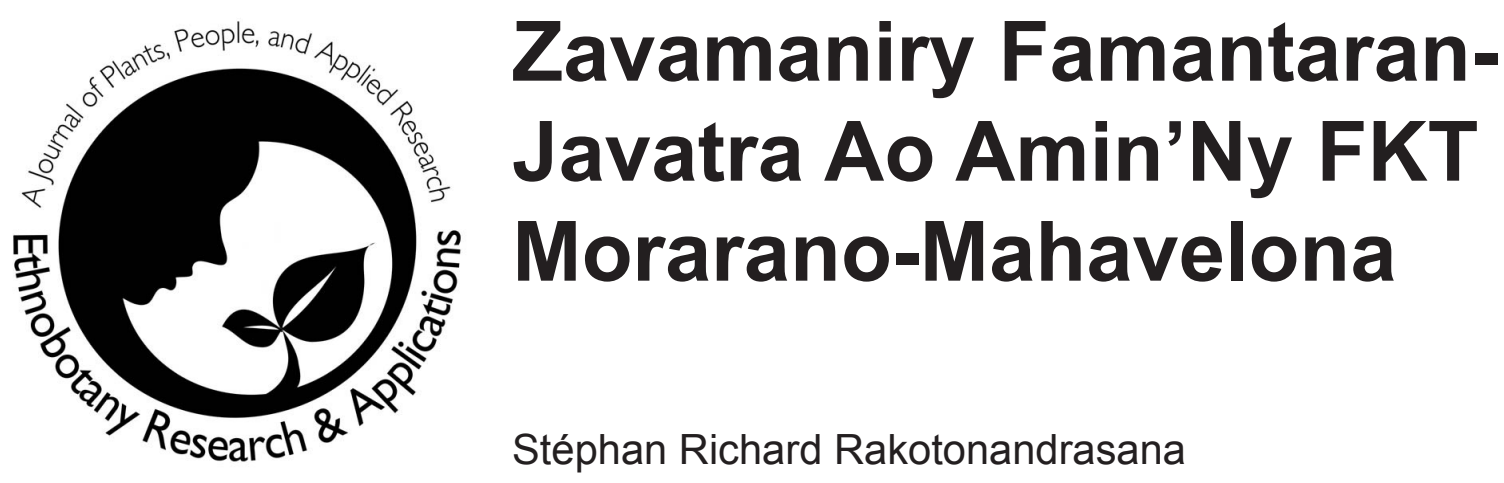

Ny tananan'i Morarano dia ao anatin'ny Kominina ambanivohitra Mahavelona, faritany Toamasina. Dimy ambinifolo kilaometatra $(15 \mathrm{~km})$ atsimo andrefan'ny renivohitry ny kaominina no misy azy. Betsimisaraka no anaran'ny foko mipetraka ao aryanisan'ny tanàna manamorona ny atialan'Analalava izy. Noho izany indrindra no antony nanaovana ny fikarohana mikasika ny fahalalàna nentimpaharazana mikasika ny zavamaniry sy ny fiaimpiainan'ny natiora izay ampiasaina amin'ny fiainana andavan'andro.

\title{
Fomba Fiasa
}

Nangonina ny olona teo an-tanàna ary nilazana ny antondia. Rehefa vita izay dia nanontaniana ny vehivavy sy lehilahy lehibe mikasika ny fahalalan'izireo momba ny zavamaniry famantarana zavatra iray na maromaro manodidina azy. Samy natao avokoa na ny fanontaniana hiarahana amin'olona maromaro na ny fanontaniana amin'ny olontokana. Avy eo nalaina ireo santionan-javamaniry izay notanisain'ny olona teo an-tanàna mba entina manamarina ny anaran'ilay zavamaniry ara-tsiansa. Rehefa tapitra ny fotoana nanaovana ny fanadihadiana dia novoriana ny olona teo an-tanàna ary novakiana teo anatrehany ny vokatra azo tamin'ny fanadihadiana mba hazahoana manampy izay tsy ampy na manintsy izay diso.

\section{Vokatry Ny Fikarohana}

Misy karazana sivy ambinifolo (19) ny zavamaniry entin'ny olona mamantatra ny zavatra manodidina azy (Tabilao1). Olona iraika ambinifolo (11) no niarahana niasa sy nametrahana ireo fanontaniana nandritry ny fanadihadiana ka dimy (5) tamin'izireo lahy ary enina (6) kosa ny vavy.

\section{Zavamaniry manambara tonon-taona}

Amin'ny faritra atsinanan'i Madagasikara dia manorana lalan-dava ny andro. Ny fiovan'ny habetsahan'ny rotsak'orana mandritry ny taona dia manambara vani-potoana roa : ny ririnina sy ny lohataona. Noho izany dia maintso lalan-dava no fijery ny atiala mandritry ny taona. Vitsy dia vitsy ny karazan-kazo manintsan-dravina. Ireo zavamaniry manitsandravina ireo no entin'ny olona hamantarana ny tonon-taona.

Rehefa mandrara-dravina ny Bonara, ny Antafana, ny Hintsina, ny Albizia, ny Aviavy, ny Sovoka dia miantomboka ny vani-potoan'ny ririnina. Rehefa manomboka mitsiry kosa ny ravin'ireo dia manomboka izay ny lohataona. Etsy andanin'izay, rehefa matoy ny voan'ny Fandramanana dia efa mieritreritra ny mponina fa ho avy ny Noely.

\section{Zavamaniry manambara fotoam-pambolena}

Vary no tena fototsakafon'ny olona amin'ity tanàna ity tahaka ny ankamaroan'ny tanàna eto Madagasikara. Noho izany ny zavamaniry mamaritra ny fotoam-pambolena ny vary no tena tantarain'ny olona. Ny zavamaniry mandrara-dravina no tena ampiasaina amin'izany.

\section{Correspondence}

Stéphan Richard Rakotonandrasana, Karoka Nasionaly Ampiharana ny Raki-Panafody (KNARP), B.P. 702, Antananarivo, MADAGASIKARA.

icbg@refer.mg

Ethnobotany Research \& Applications 3:345-347 (2005) 
Tabilao1. Famintinana famantarana lazain'ny zavamaniry

\begin{tabular}{|l|l|}
\hline Zavamaniry & Zavatra lazainy \\
\hline Fandramanana & Fiavian'ny fetin'ny Noely \\
\hline Bonara & Tonon-taona, fotoam-pambolena \\
\hline Hintsina & Tonon-taona, fotoam-pambolena \\
\hline Aviavy & Tonon-taona, fotoam-pambolena \\
\hline Antafana & Tonon-taona, fotoam-pambolena \\
\hline Bizia & Tonon-taona, fotoam-pambolena \\
\hline Savoka & Tonon-taona, fotoam-pambolena \\
\hline Sadodoka & Tonon-taona \\
\hline Rangotra & Tanety tsy mamokatra \\
\hline Ahipody & Tanety tsy mamokatra \\
\hline Tenona & Tanety tsy mamokatra \\
\hline Forombato & Konka tsy mamokatra \\
\hline Penja & Konka tsy mamokatra \\
\hline Harongana & Efa lonaka ny tany, \\
\hline taompamokarana \\
\hline Romintina & Efa lonaka ny tany \\
\hline Dingadingana & Efa lonaka ny tany \\
\hline Hasina & Fizaran-tany, Fananan-tany \\
\hline Tonona & Fisokina \\
\hline Hazomananjara & Fisokina \\
\hline & \\
\hline
\end{tabular}

Manomboka mitsiry ny ravin'ny Bonara, ny Antafana, ny Hintsika, ny Albizia, ny Aviavy, ny Sovoka dia mikarakara ny asa vary ny mponina. Misy fetrany ny fotoana fikarakarana. Rehefa tapitra mihintsana ny voan'ny sadodoka dia tapitra ihany koa ny fotoam-pambolem-bary. Tsy tokony hiasa vary intsony rehefa vaky felana ny Fontsy. Efa tokony hiteraka ny vary amin'io fotoana io.

Miaraka miantomboka amin'ny fambolena vary ny fambolena mangahazo. Rehefa manitsan-dravina ny mangahazo dia manomboka mamboly tsomanga indray ny olona.

\section{Zavamaniry milaza zavatra masina}

\section{Ny tsiny}

Ny fisian'ny vahy lehibe mifamatotra ao anaty ala dia manambara fa misy tsiny io ala io. Noho izany raha te hanao tavy io ala io ny olona dia tsy maintsy manao ny fomba fanalana ny tsiny.

Ny fisokina
Ny hazo vaventy sy mety maharitra ela manodidina ny tanana dia nosafidian'ny razana hanaovana joro na fisokina. Amin'ity tanànan'i Morarano ity dia Ampaly be no voasafidy anaovana ny fisokina. Amin'izao fotoana izao moa ny mponina ao dia efa tsy mijoro eo amin'ilay fisokina intsony noho ny fivavahana kristianina. Na izany aza anefa dia mbola tsy misy sahy mandoto ny manodidina ny toerana hijoroan'ny fisokiana io. Raha araka ny tantara dia matetika ny fisokina fefena amin'ny karazan-kazo atao hoe Hasina ary eo akaikiny volena ny hazo mitondra ny anarana hoe Hazomananjara. .

Ny fisokina iray hafa ao an-tanàna dia somary eo afovoan'ny tanana no misy azy. Hazo natsatoka izy io ary maranitra ny lohany. Tonognana (Antohiravivina) no anaran'ilay hazo atsatoka mba ho fisokiana karazany faharoa ity.. Hazo mafy tokoa izy io ary hambolena zavamaniry hafa eo amin'ny fotony. Mbola misy mangataka amin'ny razana eo aminy.

\section{Zavamaniry milaza fahalonaky ny tany}

\section{Eny an-tanety}

Aorian'ny tavy voalohany, efa lonaka ka azo tavasana fanindroany ny tanety rehefa mody atiala indray. Mody atiala ny tanety rehefa mahatratra $5 \mathrm{~m}$ farafahakeliny ny hazo maniry eo. Ireto ny karaza-kazo hita eo amin'izany : Ranomintina, Harongana, Dingadingana.

Tsy mety mahavokatra intsony ny tanety rehefa maniry eo ny ahipody sy ny Rangotra fohy. Raha Rangotra lava anefa no hita eo dia mety hamokatra ihany ilay tany saingy tapahana roa volana alohan'ny handorana azy.

\section{Eny an-kôkana}

Ny kôkana rehetra dia mahavokatra avokoa afatsy ny kôka hanirian'ny Forombato sy ny Penja.

\section{Zavamaniry mamaritra fananana}

Ety an-tanàna dia ny hasina no ataon'ny mponina fizarana tany. Izany hoe famefena ny faritry ny trano na tokotany. Eny an-tanety kosa dia hasina na Kininina no ataon'ny mponina famantarana ny faritry ny taniny. Ny fisian'ny kininina amin'ny faritra iray dia manambara fa efa manatompo ilay tany. Amin'ny ankapobeny dia hazo maharitra ela izy ireo.

\section{Zavamaniry manambara taom-pamokarana}

Tsy dia misy olona mahalala firy ity zavatra ity. Araka ny tantara, rehefa mamoa be ny Harongana sy ny Gavo dia ho tsara ny vary amin'ny taona. Torak'izany ihany koa ny Voarobary. Rehefa mamoa be izy io dia ho tsara izay ny vokatra. 


\section{S. R. Rakotonandrasana - Zavamaniry Famantaran-Javatra Ao Amin'Ny FKT 347 Morarano-Mahavelona}

Raha ireo no jerena dia hita fa mampianatra sy manoro ny olona handrindra ny fiainany ny fomba fiainan'ny voahary manodidina azy. Ny finoan'ny razana teo aloha dia mifono hevi-dalina izay arofanina misakana ny olona tsy handrava ny rindra voajanahary efa misy. Ny natiora ihany koa no zavatra voalohany ampiasain'ny olona amin'ny fifandaminana eo an-tanàna. Tandindomin-doza anefa ireny ankehitriny noho ny firoboroboan'ny kolontsaina vahiny sy ny tsy fahampian'ny fahalalana mikajy ny voahary ary ny fahapotehan'ny tontolo iainana.

FISAORANA

Isaorana eto ny:

- Sampam-pikarohana KNARP sy ny Missouri Botanical Garden (MBG) tamin'ny fikarakarana ny fikarohana

- tetikasan'ny MBG aty Analalava/Mahavelona nanamora ny fifandraisana tamin'ny fokonolona,

- ny fokonolona rehetra ao Morarano

- tetikasa International Cooperative Biodiversity Group (ICBG) nanome ny vola nanantontosana ny atrik'asa natao tao Antananarivo sy ny fikarohana natao tany Marorarano. 
